\title{
Documento
}

\section{CITOLOGÍA DE BASE LÍQUIDA: REVISIÓN DE LA HISTORIA Y LOS ESTUDIOS AL RESPECTO}

\author{
Paolo Ricci A. ${ }^{1}$, Ernesto Perucca P. ${ }^{1}$, Josip Koljanin V. ${ }^{1}$, Eduardo Baeriswyl T. ${ }^{1}$ \\ ${ }^{1}$ Servicio de Obstetricia y Ginecología, Hospital Barros Luco - Trudeau.
}

\begin{abstract}
RESUMEN
George Nicolas Papanicolaou introdujo la citología diagnóstica de frotis vaginal en 1940, técnica de tamizaje de bajo costo y de aplicación masiva. Donde se implementó, ha permitido aumentar la detección del cáncer cérvico uterino en etapas precoces y de lesiones premalignas, reduciendo significativamente la mortalidad por esta causa. El frotis tecnificado, de alto costo, en teoría resuelve los cinco problemas de la convencional: 1) captura de la totalidad de la muestra, 2) fijación deficiente, 3) distribución aleatoria de células anómalas, 4) existencia de elementos perturbadores, 5) calidad del frotis. Estudios en grandes poblaciones no evidencian diferencias significativas en su capacidad diagnóstica comparados con la citología convencional que permitan sugerir su uso en el sistema público de salud.
\end{abstract}

\section{PALABRAS CLAVES: Frotis de Papanicolaou, citología de base líquida, cáncer cérvico-uterino}

\section{SUMMARY}

Liquid-based cytology and conventional cervical smears: a completed review of the literature is discussed. Evidence based on population studies still does not support it use for a public health system.

\section{KEY WORDS: Conventional cervical smears, liquid-based cytology, cervical cytology, cervical cancer}

\section{INTRODUCCIÓN}

La introducción del frotis de células recolectadas del cuello uterino y vagina, para la detección de cáncer cérvico-uterino, se debe a George Nicolas Papanicolaou. Ha pasado largo tiempo desde que introdujo esta técnica en 1940 (1-4). Sin embargo, se trata de un método de tamizaje con algunas desventajas, que ha motivado la creación de nuevas técnicas, como es la citología de base líquida.

Siempre que se introducen nuevos exámenes, debemos ser cautos en analizar los estudios existentes, antes de implementar esas técnicas, traspasando los costos a los usuarios y servicios pú- blicos de salud. Por estos motivos, el objetivo de este estudio es analizar la capacidad diagnóstica de la citología convencional, hasta llegar a la citología de base líquida con sus respectivas ventajas y limitaciones.

\section{Frotis de Papanicolaou: ventajas y limitaciones}

El frotis de Papanicolaou es una técnica de tamizaje de bajo costo y de aplicación masiva. Gracias a su implementación, aumentó la detección del cáncer cérvico-uterino en etapas precoces disminuyendo la incidencia. Por otro lado permitió reducir significativamente la mortalidad por esa causa $(5,6)$ 
El cáncer cérvico uterino provoca alrededor de 500.000 muertes al año en el mundo. Su incidencia es de 40 por 100.000 mujeres en países en desarrollo (7). En Chile, en 2002, se diagnosticaron 980 casos nuevos, y fallecen anualmente alrededor de 628 mujeres, con una tasa de mortalidad de 8 por 100.000 mujeres. Es el cáncer con la mayor tasa de años potencialmente perdidos en la mujer (129 años por 100.000 mujeres) (8-12).

La Agency for Health Care Policy and Research publicó un meta-análisis de 84 estudios clínicos, que estableció una capacidad diagnóstica con especificidad de $98 \%$ y sensibilidad de $51 \%$ (13). Sin embargo, en la literatura se describen proporciones de falsos negativos para cáncer invasor y para neoplasias intraepiteliales, que varían entre 0 y $70 \%$ (14).

El frotis de Papanicolaou logra reunir entre 600.000 y 1,2 millones de células epiteliales cervicales y menos del $20 \%$ se transfiere al portaobjeto, el traspaso de células es aleatorio y sujeto a error si las células anormales no se distribuyen de forma homogénea por toda la muestra (14). Davey y cols en 1993 (15) y Naaryshkin (16) en 1997 publican estudios de análisis retrospectivos de cáncer cérvico uterino; en ellos demuestran que entre el 5 y $15 \%$ tenían citologías de Papanicolaou con falsos negativos, que correspondían a ASCUS y LIE de alto y bajo grado. Weintraub y Morabia en 2000, demuestran que las limitaciones de la técnica se debió a la presencia de muestras no valorables o poco significativas, estimándose que dos tercios se deben a errores en la toma de muestra (17). En tres trabajos de investigación de falsos negativos, se demostró que fueron menos comunes los errores de detección que los de muestreo (18-20).

Por otro lado la preparación del frotis es una técnica variable y mal controlada. La muestra debe fijarse rápido para evitar la desecación y la degeneración de las células; además debe extenderse de manera uniforme en toda la superficie del portaobjeto. Otro problema es la presencia de sangre y células inflamatorias que compiten por el área de superficie del portaobjeto, llegando a ocultar células epiteliales $(21,22)$.

\section{Del frotis tradicional de Papanicolaou al de base líquida}

La falta de precisión en los diagnósticos basados en citologías realizadas entre los años 1972 y 1977, a usuarios del sistema de salud cerrado de la Fuerza Aérea de los Estados Unidos, determinó una serie de demandas; el gobierno norteamericano solicitó una investigación para definir responsabilidades ante lo ocurrido. Como resultado, se dice: "el alto subdiagnóstico de las pacientes sometidas a Papanicolaou se debió a un control normativo deficiente del personal de laboratorio y a las grandes cargas de trabajo" (23). A partir de esto se comienzan a denunciar a otros laboratorios del resto de EEUU. En 1978, The Wall Street Journal denuncia a través de la publicación de un informe, a los laboratorios de citología del este de EEUU. En este artículo concluyen que el excesivo volumen y los costos bajos, sacrificaban la precisión (24). En respuesta, Ia CLIA (Clinical Laboratorios Improvements Act) en 1988, redacta la Ley para el mejoramiento de los laboratorios (25), estableciendo que: "una persona encargada de la valoración de preparaciones citológicas mediante técnica microscópica no automatizada, no debe revisar más de 100 portaobjetos en 24 horas, y que debe realizarse un mínimo de repetición de la valoración del $10 \%$ de los valorados como normales en el primer screening".

Revisiones como las de Hutchinson y cols (20), Kristensen y cols (19) y Joseph y cols (18), demostraron que las limitaciones del frotis de Papanicolaou, más que a error humano o de los laboratorios, se debían a otros factores propios del procedimiento mismo.

Metanálisis publicados por Fahey (26) y Boyko (27) mostraron que la sensibilidad del Papanicolaou convencional para la detección de precursores de cáncer cervical era menor del $50 \%$; esto fue corroborado más tarde por la Agency for Health Care Policy and Research (28).

Con el desarrollo de instrumentos computarizados y mejores ópticas, se creía que aumentaría la sensibilidad y disminuirían los falsos negativos, sin embargo, se identificaron limitaciones en el muestreo y en la preparación. En respuesta a esta problemática se crea el ThinPrep ${ }^{\mathrm{TM}}$ (Cytyc Corp, Boxborough, Massachussets) y el AutocytePrep ${ }^{\mathrm{TM}}$, llamado en sus inicios CytoRich ${ }^{\text {TM }}$ (TriPath Imaging, Inc Burlington, North California). En mayo de 1996 se aprueba el uso de Papanicolaou ThinPrep ${ }^{\mathrm{TM}}$ (Cytyc Corporation Boxborough, MA), por considerarse más efectivo para detección de NIE de bajo grado y lesiones de grados altos al compararlo con el método convencional.

\section{Frotis tecnificado: ventajas}

Al revisar los trabajos sobre frotis de capa delgada y base líquida, es posible separar los de 
muestra dividida y los de uso deseado, directo al frasco. En los de muestra dividida se obtiene el frotis que es usado para PAP convencional, y el material residual del instrumento de obtención es lavado en el medio de captación para prepararlo en capa delgada. En los de uso deseado o directo al frasco, se obtiene la muestra para colocarla directo en el medio líquido, para luego compararla con testigos históricos.

Ambas clases de estudio tienen reparos importantes, en el primero se utiliza lo que queda de la muestra en el instrumento recolector, para medio líquido; según algunos podrían quedar menos células anormales para este segundo estudio. En cambio el estudio directo puede compararse con poblaciones testigos que son totalmente dispares. Recordemos que las pacientes que tienen acceso a técnicas más caras y nuevas, son generalmente de estratos socio económicos superiores, que como sabemos tiene una epidemiología diferente. Por otro lado es necesario recordar que no existen a la fecha, trabajos randomizados prospectivos.

El frotis tecnificado en teoría resuelve los cinco problemas del Papanicolaou convencional (29): 1) captura de la totalidad de la muestra, 2) fijación deficiente, 3) distribución aleatoria de células anómalas, 4) existencia de elementos perturbadores, 5) calidad del frotis.

En esta técnica se introduce el instrumento de obtención de la muestra teóricamente en su totalidad en un medio líquido, fijando las células y evitando el degeneración por aire; posteriormente se realiza una mezcla que produce una muestra homogénea $(29,30)$. Por otro lado los problemas de inclusión de elementos perturbadores que dificultan la correcta visión, y la mala calidad del frotis representada por muestras gruesas, fue resuelta de dos maneras diferentes según ThinPrep ${ }^{\mathrm{TM}}$ y Autocyte Prep ${ }^{\mathrm{TM}}$.

Autocyte Prep ${ }^{\mathrm{TM}}$ somete la muestra a un mezclado para luego separar por capas en gradiente. Luego centrifuga separando las células inflamatorias y la sangre de las células epiteliales. Una pipeta automatizada traslada la muestra libre de elementos perturbadores, a un portaobjetos por gravedad, obteniendo una capa delgada. El ThinPrep ${ }^{\text {TM }}$ en cambio mezcla la muestra en un cilindro que posee una membrana en un extremo, por donde se aspira el medio líquido y elementos perturbadores, basado en el tamaño de los poros; luego se transfieren las células obtenidas en la membrana a un portaobjetos. En ambos se obtendrían muestras finales con 50.000 a 75.000 células, en zonas circulares.
Los PAP tecnificados serían mejores en la detección de precursores del cáncer cervical (Tablas I y II). Todos los trabajos muestran un aumento, en su gran mayoría estadísticamente significativos en la detección de lesiones intraepiteliales (LIE). Sin embargo, un estudio realizado por Takahashi en 1997 evidencia una relación inversa en la detección de LIE, a favor del convencional (31).

En las muestras procesadas por PAP tecnificado debieran disminuir los porcentajes de ASCUS, probablemente por la mejor fijación y calidad del portaobjetos. Sin embargo, algunos estudios muestran una relación favorable a la citología convencional (Tablas III y IV).

\section{Un estudio a tener presente}

Cheung y cols (51), en 2003, presentaron un estudio comparativo entre PAP convencional y tecnificado de base líquida. Para ello compararon, los datos de 191.581 PAP convencionales realizados entre el 1 de marzo de 1998 y el 28 de febrero de 2000, con 190.667 PAP de base líquida (Thin Prep), realizados entre 1 de marzo de 2000 y el 28 de febrero de 2002. Con PAP de base líquida los resultados insatisfactorios, se redujeron de $0,48 \%$ (convencional) a $0,32 \%$ (base líquida). Los considerados subóptimos (artefacto, muestra escasa, problemas de fijación, sangre, muestras

\section{Tabla I}

\section{RENDIMIENTO EN LA DETECCIÓN DE LIE EN THINPREP Y AUTOCYTE EN ESTUDIOS DE MUESTRA DIVIDIDA, COMPARADO CON PAP CONVENCIONAL}

\begin{tabular}{lrcc}
\hline Estudio & Casos & $\begin{array}{c}\text { Conven- } \\
\text { cional (\%) }\end{array}$ & $\begin{array}{c}\text { ThinPrep } \\
\text { (\%) }\end{array}$ \\
\hline Lee (32) & 6.747 & 8,0 & 9,4 \\
Roberts (33) & 35.560 & 2,0 & 2,3 \\
Corkill (34) & 1.583 & 2,7 & 5,6 \\
Wang (35) & 972 & 4,4 & 6,0 \\
Shield (36) & 300 & 7,0 & 8,3 \\
Hutchinson (29) & 8.636 & 4,9 & 5,2 \\
\hline Estudio & Casos & Conven- & AutocytePrep \\
& & cional (\%) & $(\%)$ \\
\hline Vassilakos (37) & 560 & 3,8 & 4,6 \\
Takahashi (31) & 2.000 & 3,5 & 3,4 \\
Wilbur (38) & 286 & 4,2 & 9,1 \\
Kunz (39) & 554 & 1,4 & 3,4 \\
Bishop (21) & 8.983 & 5,2 & 5,9 \\
Minge (40) & 14.539 & 4,4 & 5,8 \\
\hline
\end{tabular}


Tabla II

RENDIMIENTO EN LA DETECCIÓN DE LIE EN THINPREP Y AUTOCYTE EN ESTUDIOS DIRECTO AL
FRASCO COMPARADO CON PAP CONVENCIONALES HISTÓRICOS

\begin{tabular}{|c|c|c|c|c|}
\hline \multirow[b]{2}{*}{ Estudio } & \multicolumn{2}{|c|}{ Casos } & \multicolumn{2}{|c|}{$\%$ LIE } \\
\hline & Convencional & ThinPrep & Convencional & ThinPrep \\
\hline Weintraub (41) & 13.067 & 18.247 & 1,0 & 2,9 \\
\hline Bolick (22) & 39.408 & 10.694 & 1,1 & 2,9 \\
\hline Papillo (42) & 18.569 & 8.541 & 1,6 & 2,5 \\
\hline Dupree (43) & 22.323 & 19.351 & 1,2 & 1,7 \\
\hline Carpenter (44) & 5.000 & 2.727 & 7,7 & 10,5 \\
\hline Guidos (45) & 5.423 & 9.583 & 1,3 & 4,7 \\
\hline Díaz-Rosario (46) & 74.756 & 56.339 & 1,8 & 3,2 \\
\hline Weintraub (17) & 129.619 & 39.455 & 0,6 & 2,3 \\
\hline Estudio & Convencional & AutocytePrep & Convencional & AutocytePrep \\
\hline Vassilakos (47) & 15.402 & 32.655 & 1,1 & 3,6 \\
\hline Vassilakos (48) & 88.569 & 111.358 & 2,0 & 3,2 \\
\hline Vassilakos (49) & 19.923 & 81.120 & 1,2 & 3,4 \\
\hline Tench (50) & 10.367 & 2.231 & 1,0 & 1,7 \\
\hline
\end{tabular}

Tabla III

DETECCIÓN DE ASCUS EN ESTUDIOS DIRECTO

AL FRASCO EN THINPREP Y AUTOCYTE, COMPARADO CON PAP CONVENCIONAL

\begin{tabular}{lcccc}
\hline \multirow{2}{*}{ Estudio } & Convencional & Casos & & \multicolumn{2}{c}{ \% ASCUS } \\
\hline Weintraub (41) & 13.067 & ThinPrep & Convencional & ThinPrep \\
Bolick (22) & 39.408 & 18.247 & 1,6 & 2,7 \\
Papillo (42) & 18.569 & 10.694 & 2,3 & 2,9 \\
Dupree (43) & 22.323 & 8.541 & 9,0 & 4,6 \\
Carpenter (44) & 5.000 & 19.351 & 4,9 & 6,6 \\
Guidos (45) & 5.423 & 2.727 & 3,4 \\
Díaz-Rosario (46) & 74.756 & 9.583 & 2,0 & 4,5 \\
Weintraub (17) & 129.619 & 56.339 & 4,8 & 2,4 \\
\hline Estudio & Convencional & AutocytePrep & 1,5 & AutocytePrep \\
\hline Vassilakos (47) & 15.402 & 32.655 & Convencional & 1,6 \\
Vassilakos (48) & 88.569 & 111.358 & 3,7 & 1,2 \\
Vassilakos (49) & 19.923 & 81.120 & 3,0 & 1,9 \\
Tench (50) & 10.367 & 2.231 & 3,5 & 5,5 \\
\hline
\end{tabular}

inadecuadas) se redujeron de $19,1 \%$ (convencional) a $12,9 \%$ (base líquida). La detección de carcinoma de células escamosas, adenocarcinoma y neoplasias intraepiteliales de alto grado, no cambiaron significativamente en el grupo de base líquida. Sin embargo, aumentó la detección de células escamosas atípicas de significado indeterminado (ASCUS) $(3,19 \% \mathrm{v} / \mathrm{s} 3,74 \%)$, la determinación de células glandulares atípicas de significado indeterminado (ASGUS) $(0,07 \% \mathrm{v} / \mathrm{s} \quad 0,09 \%)$, los NIE de bajo grado $(1,01 \% \mathrm{v} / \mathrm{s} 1,67 \%)$, y la detección de Actinomices (0,52\% v/s 1,07\%) (proporción similar de usuarias de DIU). Es el trabajo de mayor casuística en citología de base líquida; al analizar la conclusión de los autores, hay que tener presente que muchas de las diferencias no fueron significativas. Debemos tener presente bajo los resultados globales, los costos de implemen- 
Tabla IV

\section{DETECCIÓN DE ASCUS EN PAP CONVENCIONAL, COMPARADO CON ESTUDIOS DE MUESTRA DIVIDIDA EN AUTOCYTEPREP}

\begin{tabular}{lrrcc}
\hline Estudio & Año & Casos & $\begin{array}{c}\text { Conven- } \\
\text { cional (\%) }\end{array}$ & $\begin{array}{c}\text { Autocyte } \\
\text { Prep (\%) }\end{array}$ \\
\hline Vassilakos (37) & 1996 & 560 & 12,9 & 7,7 \\
Takahashi (31) & 1997 & 2.000 & 1,1 & 4,6 \\
Wilbur (38) & 1997 & 286 & 13,6 & 13,3 \\
Kunz (39) & 1998 & 554 & 9,6 & 3,3 \\
Bishop (21) & 1998 & 8.983 & 6,2 & 6,0 \\
Minge (40) & 2000 & 14.539 & 6,9 & 5,9 \\
\hline
\end{tabular}

tación versus los beneficios de la técnica. Por otro lado resulta importante analizar la implementación en el sector público, ¿se justifica a la luz de lo anterior?.

\section{CONCLUSIONES}

Como en toda nueva técnica que se aplica en medicina debemos ser cautos, y revisar la literatura existente al respecto. No se trata de evitar el desarrollo de nuevas técnicas en nuestra práctica diaria profesional, pero sí ser lo suficientemente críticos para saber cuándo y dónde implementar las nuevas tecnologías. De lo contrario podemos afectar a nuestros pacientes y a los servicios de salud públicos, al traspasar los costos de una apresurada implementación. No existen estudios prospectivos randomizados, y que tanto los directos al frasco, como los de muestra dividida son criticables metodológicamente.

En Chile, el costo de la máquina para citología líquida es de 50.000 dólares, mientras que sólo los insumos para cada toma de muestra suman alrededor de 6 dólares. En cambio el costo total de una citología convencional en el sistema público de salud, no supera los 2,5 dólares. Debemos tener presente además que los países que han incorporado la citología líquida, como tamizaje sobre la citología convencional, poseen realidades económicas superiores y sistemas de salud basados en su gran mayoría en seguros médicos.

Por todo lo anterior no parece justificable la implementación de esta nueva técnica para los usuarios del sistema de salud pública, por no tener claras ventajas, y sobretodo considerando los costos de implementación y por examen.

Una optimización de la citología convencional debe considerar mejoras en los implementos de toma de muestras (cepillos y espátulas plásti- cas), sin aumentar los costos, para continuar siendo una técnica de tamizaje de bajo costo y sencilla.

\section{BIBLIOGRAFÍA}

1. Vilos GA. After office hours. The history of the Papanicolau smear and the odyssey of George and Andromache Papanicolaou. Obstet Gynecol 1998; 91: 47983.

2. Berkow S.G. After Office Hours. A visit with Dr. George N. Papanicolaou. Obstet Gynecol 1960; 16: 243-52.

3. Papanicolaou GN, Traut HF. Diagnosis of uterine cancer by the vaginal smear. NY: Commonwealth Fund, 1943.

4. Carmichael DE. The Pap Smears: Life of George N. Papanicolaou. Springfield, Illinois, 1973.

5. Quinn M, Babb P, Jones J, Allen E. Effect of screening on incidence of and mortality from cervical cancer in England: evaluation based on routinely collected statistics. BMJ 1999; 318: 904-8.

6. MacGregor JE, Campbello MK, Mann EM, Swanson KY. Screening for cervical intraepithelial neoplasia in north east Scotland shows fall in incidence and mortality from invasive cancer with concomitant rise in preinvasive disease. BMJ 1994; 308: 1407-11.

7. Franco EL, Duarte-Franco ED, Ferenczy A. Cervical cancer: epidemiology, prevention and the role of human papillomavirus infection. CMAJ 2001; 164(7): 1017-25.

8. Suárez E, Prieto M, Rojas I, Fernández B, Prado R, Olfos P. Programa nacional de cáncer cervicouterino. Rev Chil Obstet Ginecol 2001; 66(6): 480-91.

9. Serman F. Cáncer cérvico uterino: epidemiología, historia natural y rol del virus papiloma humano, perspectivas en prevención y tratamiento. REV CHIL OBSTET GINECOL 2002; 67(4): 318-23.

10. Instituto Nacional de Estadísticas de Chile. MINSAL, 2000.

11. Ministerio de Salud de Chile. Orientaciones para pesquisa y control del cáncer cérvico uterino.

12. Comisión Nacional del Cáncer cérvico-uterino. Ministerio de Salud. Diagnóstico y tratamiento del cáncer cérvico-uterino. Documento de trabajo, abril de 2004.

13. Hutchinson ML, Isenstein LM, Goodman A, Hurley AA, Douglass KL, Mui KK, Patten FW, Zahniser DJ. Homogeneous sampling accounts for the increased diagnostic accuracy using the ThinPrep Processor. Am J Clin Pathol 1994;101(2): 215-9.

14. Soost HJ, Lange HJ, Lehmacher W, Ruffing-Kullmann B. The validation of cervical cytology. Sensitivity, specificity and predictive values. Acta Cytol 1991; 35(1):8-14.

15. Davey DD, Nielsen ML, Frable WJ, Rosenstock W, Lowell D, Kraemer BB. Improving accuracy in gynaecologic cytology: results of the College of American Pathologists interlaboratory comparison program in 
cervicovaginal cytology. Arch Pathol Lab Med 1993; 117: 1193-8.

16. Naryshkin S. The false negative fraction for Papanicolaou smears: how often are "abnormal" smears not detected by "Standard screening cytologist?" Arch Pathol Lab Med 1997; 121: 270-2.

17. Weintraub J, Morabia A. Efficacy of a liquid-based thin layer method for cervical cancer screening in a population with a low incidence of cervical cancer. Diagn Cytopathol 2000; 22(1): 52-9.

18. Joseph MG, Cragg F, Wright VC, Kontozoglou TE, Downing P, Marks FR. Cyto-histologycal correlates in a colposcopic clinic: A 1 year prospective study. Diagn Cytopathol 1991; 7(5): 477-81.

19. Kristensen GB, Skyggebjerg KD, Holund B, Holm K, Hansen MK. Análisis of cervical smears obtained within three years of the diagnosis of invasive cervical cancer. Acta Cytol 1991(1); 35:47.

20. Hutchinson ML, Bassin CM, Ball HG. The efficacy of an automated preparation device for cervical cytology. Am J Clin Pathol 1991; 96:300-5.

21. Bishop JW, Bigner SH, Colgan TJ, Husain M, Howell LP, Mclntosh KM, Taylor DA, Sadeghi MH. Multicenter masked evaluation of AutoCyte PREP thin layers with matched conventional smears. Including initial biopsy results. Acta Cytol 1998; 42(1): 189-97.

22. Bolick DR, Hellman DJ. Laboratory implementation and efficacy assessment of the ThinPrep cervical cancer screening system. Acta Cytol 1998; 42(1): 209-13.

23. New Papanicolaou test suggested for Air Force dependents. US Med 1978: 1-3.

24. Bogdanich W. Lex Laboratories: The Pap test misses much cervical cancer through labs'errors. The Wall Street Journal, November 11,1987.

25. Clinical Laboratory Improvement Amendments of 1988 (CLIA 88). Public Law 100-578. Fed Register 1990; 55: 953.

26. Fahey MT, Irwig L, Macaskill P. Meta-analysis of Pap test accuracy. Am J Epidemiol 1995; 141(7): 680-9.

27. Boyko EJ. Meta-analysis of Pap test accuracy. Am J Epidemiol 1996; 143(4): 406-7.

28. Agency for Health Care Policy and Research: Evidence Report/Technology Assessment № 5. Evaluation of Cervical Cytology. 1999. Report № 99E010.

29. Hutchinson ML, Zahniser DJ, Sherman ME, Herrero $R$, Alfaro M, Bratti MC, Hildesheim A, Lorincz AT, Greenberg MD, Morales J, Schiffman M. Utility of liquid-based cytology for cervical carcinoma screening: results of a population-based study conducted in a region of Costa Rica with a high incidence of cervical carcinoma. Cancer 1999; 87(2): 48-55.

30. Koss LG. Utility of liquid-based cytology for cervical carcinoma screening. Cancer 2000; 90(1): 67-9.

31. Takahashi M, Naito M. Application of the CytoRich monolayer preparation system for cervical cytology. A prelude to automated primary screening. Acta Cytol 1997; 41(6): 1785-9.
32. Lee $K R$, Ashfaq $R$, Birdsong GG, Corkill ME, Mclntosh KM, Inhorn SL. Comparison of conventional Papanicolaou smears and a fluid-based, thinlayer system for cervical cancer screening. Obstet Gynecol 1997; 90(2): 278-84.

33. Roberts JM, Gurley AM, Thurloe JK, Bowditch R, Laverty CR. Evaluation of the ThinPrep Pap test as an adjunct to the conventional Pap smear. Med $\mathrm{J}$ Aust 1997; 167(9): 466-9.

34. Corkill ME, Knapp D, Hutchinson ML. Improved accuracy for cervical cytology with the ThinPrep method and the endocervical brush spatula collection procedure. J Lower Fem Gen Tract 1998; 167: 466-69.

35. Wang TY, Chen HS, Yang YC, Tsou MC. Comparison of fluid-based, thin-layer processing and conventional Papanicolaou methods for uterine cervical cytology. J Formos Med Assoc 1999; 98(7): 500-5.

36. Shield PW, Nolan GR, Phillips GE, Cummings MC. Improving cervical cytology screening in a remote, high risk population. Med J Aust 1999; 170(6): 2558.

37. Vassilakos P, Cossali D, Albe X, Alonso L, Hohener $\mathrm{R}$, Puget E. Efficacy of monolayer preparations for cervical cytology: emphasis on suboptimal specimens. Acta Cytol 1996; 40(3): 496-500.

38. Wilbur DC, Facik MS, Rutkowski MA, Mulford DK, Atkison KM. Clinical trials of the CytoRich specimenpreparation device for cervical cytology. Preliminary results. Acta Cytol 1997; 41(1): 24-9.

39. Kunz J, Rondez R, Yoshizaki C, Fivian M, Held G, Lind B. Comparison of conventional PAP smears with thin layer specimen (liquid-based PAP test) and correlation with cytopathological findings with HPV status using the hybrid capture system Schweiz Rundsch Med Prax 1998; 87(43): 1434-40.

40. Minge L, Fleming $M$, VanGeem T, Bishop JW. AutoCyte Prep system vs. conventional cervical cytology. Comparison based on 2,156 cases. J Reprod Med 2000; 45(3): 179-84.

41. Weintraub J. The coming revolution in cervical cytology: A pathologist's guide for the clinician. Ref Gynecol Obstet 1997; 5: 2-6.

42. Papillo JL, Zarka MA, St John TL. Evaluation of the ThinPrep Pap test in clinical practice. A sevenmonth, 16,314-case experience in northern Vermont. Acta Cytol 1998; 42(1): 203-8.

43. Dupree WB, Suprun HZ, Beckwith DG, Shane JJ, Lucente V. The promise and risk of a new technology: The Lehigh Valley Hospital's experience with liquid-based cervical cytology. Cancer 1998; 84(4): 202-7.

44. Carpenter AB, Davey DD. ThinPrep Pap Test: performance and biopsy follow-up in a university hospital. Cancer 1999; 87(3): 105-12.

45. Guidos BJ, Selvaggi SM. Use of the Thin Prep Pap Test in clinical practice. Diagn Cytopathol 1999; 20(2): 70-3

46. Diaz-Rosario LA, Kabawat SE. Cell block prepa- 
ration by inverted filter sedimentation is useful in the differential diagnosis of atypical glandular cells of undetermined significance in ThinPrep specimens. Cancer 2000; 90(5): 265-72.

47. Vassilakos P, Griffin S, Megevand E, Campana A. CytoRich liquid-based cervical cytologic test. Screening results in a routine cytopathology service. Acta Cytol 1998; 42(1): 198-202.

48. Vassilakos P, Saurel J, Rondez R. Direct-to-vial use of the AutoCyte PREP liquid-based preparation for cervical-vaginal specimens in three European laboratories. Acta Cytol 1999; 43(1): 65-8.
49. Vassilakos P, Schwartz D, de Marval F, Yousfi L, Broquet G, Mathez-Loic F, Campana A, Major A. Biopsy-based comparison of liquid-based, thin-layer preparations to conventional Pap smears. J Reprod Med 2000; 45(1): 11-6.

50. Tench W. Preliminary assessment of the AutoCyte PREP. Direct-to-vial performance. J Reprod Med 2000; 45(11): 912-6.

51. Cheung AN, Szeto EF, Leung BS, Khoo US, Ng AW. Liquid based cytology and convencional cervical smears Cancer 2003; 99(6): 331-5. 